\title{
Dr. Unnas Dermatologische Preisaufgabe für 1900
}

\section{lauie $3 / 4$ :}

Es soil untersucht werden:

„Die feinere Apchitektup dep ppimäpen Haut-capcinome und insbesondere die bei ihnen obwaltenden vepschiedenen Beziehung en zwisehen Epithelwuchepung · und

Bindeg.ewebswidepstand."

Die Bewerbung ist unbeschränkt. Der Pi eis beträgt M. 300,-- Die Arbeit ist bis Anfang Dezember 1900 bei der Verlagsbuch-handlung Leopold Voss in Hamburg, Hohe Bleiclien 34, einzu-reichen. Sie ist rait einem Kennwort (Motto), zu versehen; das gleicbe Kennwort ist a ff der Hülle eines beizulegenden Briefes, welcher Namen und Adresse des Yerfassers entliält, anzubringen.

Die Herren Proff. Hauser (Erlangen), Nauwerck (Chemnitz) and Ortla (Göttingen) haben es gtitigst übernommen, die ein-laufenden Arbeiten zu prüten.

Nãhe're Mitteiluogen über das Them a:

In den letzten. Jahren sind mehrfache. Untersuchungen veröffentlicht worden über die gegenseitige Abhängigkeit der beim carcinomatösen Prozess in Wucherung geratenden Gewebselemente, ohne dass dabei der Vielseitigkeit der Carcinomtypen gebührend Rechnung getragen wurde. Die Beantwortung dei*1 diesjährigen Préisaufgabe ist bestimmt, diese Lücke auszufüllen. Das Studium der unter einander so äusserst verschiedenen Hautcarcinome erscheint am geeignetsten, um bald ein grösseres Material von leicht übersehbaren und einfach zu deutenden histologischen Thatsachen zusammeu zu tragen über die Frage, unter wólehen verschiedenen Formen der earcinomatöse Prozess auf ein und denselben Gewebsboden zu wirken vermag. Ein solches· Material wird dann erst die Grundlage zu einer gerechten Würdigung der beim Carcinom in Betracht kommenden treibenden Kräfte abgeben können. Der Erkennung der Gewebsmeohanik muss beim Carcinom, wie uberall, die genaueste Kenntnis der Gewèbsarchitektur vorausgehen.

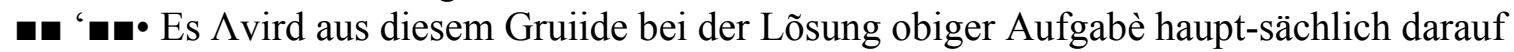
$\Lambda$ Vert .gelegt, dass ein möglichst verschiedenarti ges Material yon Hautcarqinom, eii. einer ,mit den besten Färbemethoden aus-geführten sehr genauen Untersuchung unterzogen wird.

$\mathrm{Ob}$ sich der Verfassér dàbeï dër von Unna in seiner Histopathologie gegebenen Einteilung der Hautcarcinome áns $1 / 8$ hlíesst oder nicht, ist $\Lambda$ veniger von Bedeutùng, als das, s er selbst zu einer rationellen lîinteilứg seines Materials nach $\mathrm{hi}>$ tologisehen $\operatorname{Pr}^{1} / 8$ zipien gelangt.

Selbstverständlích müssen zur exakten Darstellupg der Epi thelbin d e-gewebsgrenze die be $1 / 8$ ten Färbemethoden fur KoIГagen und Elastin, beim Studium der Epithelien und Bindegewebszelleu diejenigen für Prótoplasma herangezogen werden, da die gebräuchlichen Kernfärbungsmethoden wohl gute Uebersichtsbilder liefern, aber in alien feineren histologischen Fragen im Stiche lassen.

Beim Epithel ist ein Punkt von grosster Wichtigkeit: die scharfe ünterscheidung von wabrhaft alveolärem und Moss retikulärom Epithel wach stum, welche ihrerseits das Studium der 
Epithelfaserung und Mitosen verlangt. in Bezug auf letztere ist das Augenmerk speziell auf ihre Verteilung zu richten, zu deren Untersuchung schon Alkohol-material, selbst älteres, dienen kann. Frisches Gewebe erlaubt partielle Fixieruog in Flemming's Lösung, deren Einwirkung aber bekanntlich ein tinktorielles Studium des Protoplasmas ausschliesst. Endlich ist auch eine Untersuchung der hyalinen Epitheld egeneration und ihrer Beziehung zur Bildung von Bor $\pi$ perlen erwünscht.

In der Catis verdient besondere. Aufmerksamkeit das Vorhandenseiẁ von Plasmazellen und ihr Verhältnis zu den übrigen Bindegewebszellen, da in manchen Arbeiten der letzten Jahre das ausgedehnte Vorkommen dieser Zellen besonders im gutartigen Stadium der Carcinome ganz über4 sehen wurde. In zweiter Linie ist auf die mittelst*der neueren Färbemethoden zu erførscft $\theta$ nde Degeneration des Kollagens nnd Elastin* zu achten. 